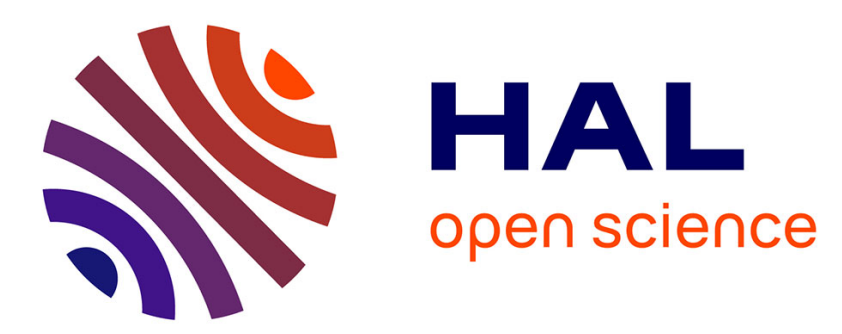

\title{
iProprio: a Smartphone-based system to measure and improve proprioceptive function
}

\author{
Quentin Mourcou, Anthony Fleury, Bruno Diot, Nicolas Vuillerme
}

\section{To cite this version:}

Quentin Mourcou, Anthony Fleury, Bruno Diot, Nicolas Vuillerme. iProprio: a Smartphone-based system to measure and improve proprioceptive function. 38th IEEE International Conference of the Engineering in Medicine and Biology Society, IEEE, Aug 2016, Orlando, FL, United States. pp.26222625. hal-01353210

\author{
HAL Id: hal-01353210 \\ https://hal.science/hal-01353210
}

Submitted on 10 Aug 2016

HAL is a multi-disciplinary open access archive for the deposit and dissemination of scientific research documents, whether they are published or not. The documents may come from teaching and research institutions in France or abroad, or from public or private research centers.
L'archive ouverte pluridisciplinaire HAL, est destinée au dépôt et à la diffusion de documents scientifiques de niveau recherche, publiés ou non, émanant des établissements d'enseignement et de recherche français ou étrangers, des laboratoires publics ou privés. 


\title{
iProprio: a Smartphone-based system to measure and improve proprioceptive function
}

\author{
Q. Mourcou, A. Fleury*, Member, IEEE, B. Diot and N. Vuillerme
}

\begin{abstract}
It is well known that proprioception is necessary to maintain balance control. Although proprioceptive exercices have shown to have beneficial effects on balance, the joint position sense is not easy to measure and patient cannot practise self-measurement. This article proposes a new system specifically desinged to allow measure and improve proprioceptive function. This so-called 'iProprio' system uses inertial sensors included in a smartphone, wireless communication and voice command to allow joint position sense measurment in autonomous way and provide a configurable vibrotactile biofeedback for end-users at home. A proof-of-concept study was performed to assess the effectiveness of iProprio on sixteen young healthy subjects. Results showed that they were able to take advantage of vibrotactile biofeedback to increase knee joint repositioning accuracy and consistency. Results suggest that iProprio can be used for rehabilitation proprioceptive home exercices.
\end{abstract}

Index Terms-Ambient Assisted Living, Biofeedback, Inertial motion unit, mHealth, Proprioception, Smartphone, Wearable device

\section{INTRODUCTION}

$\mathrm{M}$ OVING, while not thinking, seems very natural. Actually, this is allowed by proprioception, which can be defined as the perception of the position and of the movement of various body parts in space. A major component of proprioception, named Joint Position Sense (JPS), is relative to the awareness of the position of the members or segments against each other [1]. Proprioception is necessary to maintain balance, control and guide voluntary movements or evaluate the shape of an object. The dynamic part of proprioception is the kinaesthesia, defined as the sensation of the motion to locate the different parts of the body and to evaluate their movement (velocity and direction). In contrast, static part, named statesthesia, is the awareness of the position of the members or segments against each other (the JPS). Alteration of joint positition sense due to traumatic events, surgery or normal ageing may lead to specific rehabilitation to prevent injuries and reduce

Q. Mourcou is with Univ. Grenoble-Alpes, EA AGEIS, La Tronche, France, with Univ. Lille, F-59000 Lille and with Mines Douai, URIA, F59508 Douai, France (e-mail: qmourcou@gmail.com).

A. Fleury is with Univ. of Lille, F-59000 Lille and with Mines Douai, URIA, F-59508 Douai, France (e-mail: anthony.fleury@mines-douai.fr).

B. Diot is with Univ. Grenoble-Alpes, EA AGEIS, La Tronche, France and with IDS, Montceau-les-Mines, France (e-mail: b.diot@idsassistance.com).

N. Vuillerme is with Univ. Grenoble-Alpes, EA AGEIS, La Tronche, France and with Institut Universitaire de France (e-mail: nicolas.vuillerme@agim.eu). imbalance and risk of falling. Indeed, it was shown that proprioception is more important than vision in the maintenance of balance for elderly and a decrease in proprioception increases the risk of falling [2][3]. Moreover, proprioceptive physical activities have benefical effects on balance control in elderly [4]. Training proprioceptive abilities allows to maintain motor schemes as activity of the sensorimotor loop and to deepen body consciousness.

Assessing JPS consists in different exercises of joint position matching during which the patient is asked to get back to a specified angular position from a neutral one without using visual information. Different factors are likely to influence performances in such a test (e.g. time spent in the expected position, [5]) and it has been shown that passive matching from such range of motion measurement is more difficult to measure reliably than the active matching [6]. Such a test is usually performed by a physiotherapist using goniometer to measure joint angle. Recently, new measurement tools have appeared with the noteworthy particularity to be based on an unavoidable object of daily living: a smartphone. Smartphones have led to significant improvements in healthcare systems [7][8]. They have the advantage to be small, easy-to-use, affordable, connected and to include an inertial motion unit (IMU) made of 3Daccelerometer, magnetometer and gyroscope as standard. These built-in inertial sensors allow to detect and monitor both linear and angular displacements of the phone or its carrier. Particularly, it may be used to assess joint angle measurement. The current solutions are however only dedicated to measurement and none of them make use of its communicating feature to provide a feedback to the user.

The scientific contribution of this paper relies on the fact that the proposed Smartphone-based biofeedback system uses the Smartphone as an all-inclusive device to measure angle from the output of the IMU, to assess JPS and provide biofeedback to both the user and/or the clinician. A multimodal interface allows the user to choose between different sensory modalities (visual, auditory or vibrotactile) and standardized proprioceptive training protocols. This application may be used autonomously, with voice command, by the user for self-measurement, training or for rehabilitation of his proprioception at different joints at home (e.g. neck, trunk, shoulder, elbow, knee). The present paper describes the design, development and a proof-ofconcept of the vibrotactile mode of iProprio for knee proprioceptive function. 
$38^{\text {th }}$ International Conference of the IEEE Engineering in Medicine \& Biology Society, Aug. 16-19 2016, Orlando pp. $2622-2625$.

In the following, section II discusses related works on smartphone-based proprioceptive system. Section III describes the iProprio architecture. Section IV presents a proof-of-concept study to assess the effectiveness of the system as a measurement and rehabilitation device. Section $\mathrm{V}$ draws conclusions and directions to extend this work.

\section{RELATED WORKS ON SMARTPHONE-BASED SYSTEM FOR PROPRIOCEPTION}

Joint goniometry is a common mode of clinical rehabilitation. It involves propriocetive sensations derived from mechanoreceptors in the muscle, joint capsule, tendon, ligaments and skin. Differents methods can be defined in order to evaluate joint goniometry: visual evaluation, mechanical goniometer, angulation measured from images, radiographic evaluation, navigation systems, electrogoniometry or dynamometer/angular motion chairs. These technics can be used for active or passive reproduction of movement. Recent projects used Smartphone as a novel method for goniometry. Four apps were extracted from the clinical literature: (1) Simple Goniometer [9], (2) Angle [10], (3) Clinometer [11] and (4) DrGoniometer [12].

Simple Goniometer was validated on 5 asymptomatic subjects for the assesment of passive knee flexion range in comparaison with long-armed goniometer [9]. The application mimics the design of goniometer to measure, in degrees, through the built-in accelerometer, the knee range of motion measures in the supine position. Angle, which also reproduces the function of a standard goniometer, was used for the inclination of the acetabular cup during total hip arthroplasty in surgery [10]. It can calculate angles, using accelerometers, in respect to gravity, for all planes. There was less than $5 \%$ variation between these measurements and the author concluded that Smartphone is quick and accurate for acetabular placement. Clinometer uses the same principle as Angle with more features like an advanced four-way calibration. It also uses the gyroscope. Clinometer was studied for measurement of shoulder joint active and passive movements [11]. Measurements were compared with goniometer and applied on 41 patients with affected shoulders during the same day. DrGoniometer tries to reproduce image-recorded angulation in the Smartphone embedded platform. It aims at simplifying the procedure of knee joint angle measurement [12] and elbow joint angle measurement. The principle is to take a picture with the device position on the perpendicular line to the ground. Once the photo is saved, red cursors can be dragged and positioned on the body part to measure the angle.

These studies concluded that the Smartphone is a reliable and precise way to evaluate joint angles. We also performed a study, on a robot, to measure the exact performances of the smartphone [17]. Our results showed also the very good performances of smartphones. The four applications used cannot be employed by the patient alone. Moreover, the only feedback the patient may have about his performance is provided by the care provider leading the test.

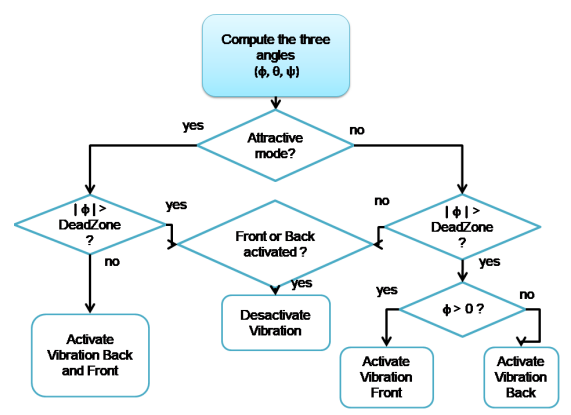

Fig. 1. Algorithm for BF generation. First, calculate the angle sensors, then check the BF mode and compare the antero-posterior angle to the value of the Dead Zone to decide whether the vibrator is activated or not.

\section{IPROPRIO SYSTEM ARCHITECTURE}

iProprio is a Smartphone-based application which can be used by both clinician and patients. Smartphone can communicate with connected objets like headphones, vibrotactile devices or smartwatch and their main advantage is to include a full IMU that can be used by any developer. iProprio tranforms the smartphone into a proprioceptive testing and training device which can be used as a rehabilitation tool for autonomous use at home. Member of the rehabilitation team such as medical doctors, physiotherapists, podiatrists and nurses can use it to quantitatively and objectively evaluate, ponctually or for long term, proprioceptive capabilities. This allows them to learn about individual information and to keep history during all the rehabilitation program. Information can be stored into the local platform and studied during medical examinations or can be transferred to the rehabiliation team via a secure server.

This section describes : (A) the sensory input unit, and (B) angle estimation and algorithm for Biofeedback (BF).

\section{A. Sensory input unit}

The sensing unit used is a smartphone (Galaxy Nexus S, Samsung, South Corea) equipped with 3 distinct MEMS sensors: (1) a 3D accelerometer (BMA220, Bosch) mostly sensing linear acceleration and providing information on orientation during static periods; (2) an integrated 3D gyroscope (MPU-3050, InvenSens), measuring angular velocity; and (3) a 3D magnetometer (YAS530, Yamaha) providing information on the orientation of the device comparing to the Earth magnetic. These 3 sensors form a 3D axes system (Fig. 2). The vibrotactile sensing unit used is a buzzband mb20 (Movon, South Corea), originally included in a rubber strap $(4 \mathrm{~cm} \times 1.8 \mathrm{~cm} \times 0.9 \mathrm{~cm}, 23 \mathrm{~g})$ that can communicate with out application via Bluetooth.

\section{B. Angle estimation and algorithm for vibrotactile $B F$ generation}

From both accelerometer and magnetometer, we can compute inclination and rotation matrices, transforming a vector from the device coordinate system to the world's coordinate system, and finally compute the orientation as tilt angles $(\theta$ and $\varphi)$ and heading $(\psi)$. The Dead Zone (DZ) is 
$38^{\text {th }}$ International Conference of the IEEE Engineering in Medicine \& Biology Society, Aug. 16-19 2016, Orlando pp. $2622-2625$.

considered to be the area within where the leg (or trunk, shoulder...) sways while the participant tries to reach the specificied angle target. This size is set to $1^{\circ}$.

Two modes can be provided for cuing with vibrotactile BF: (1) attractive and (2) repulsive. The Repulsive mode has been tested in this study. Naturally, future experimentation will compare both modes. Fig. 2 illustrates the repulsive vibrotactile $\mathrm{BF}$. While pitch angle exceeded the DZ, the front vibrator (on the tibia) is activated. Inversely, while the pitch angle was under the DZ, the back vibrator (on gastrocnemius muscle in this study) is activated. Angular data are saved in a CSV file. It can be noticed that the software is implemented to propose both attractive and repulsive $\mathrm{BF}$ generations and $\mathrm{DZ}$ size is easily parametrized.

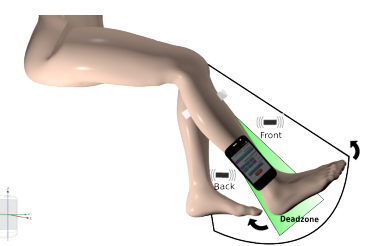

Fig. 2. Description of the Repulsive mode of vibrotactile biofeedback: the participant is asked to "move away from the vibration" until reaching the Dead Zone. Vibrators are activated outside and the location of the vibration indicates to the participant to move in the opposite direction.

\section{EXPERIMENTS AND RESULTS}

\section{A. Participants}

Ten young healthy subjects (age: $21.8 \pm 0.8$ years, mean \pm standard deviation) voluntered. None of them had history of sensory and/or motor problem, neurological disease or disorder. Participants gave written consent for their participation. Experimental procedure followed the Helsinki Declaration and was approved by the local ethics committee.

\section{B. Experimental protocol}

The test used to assess knee joint position sense comes from a standardized protocol named "active ipsilateral matching". It is commonly used and accepted in clinical routine [13]. The protocol is one of many other exercices that are embbeded in the iProprio application and for which instructions for execution are vocalized (so that protocol can be performed in an autonomous way). Each participant is seated at the edge of a table, to the level of popliteal hollow, tibial segment in a vacuum, eyes closed. He is based on upper limbs to balance and standardize his position (neutral position). Then, he is asked to extend his leg to the specified angle, 20 degrees from his neutral position, without time constraint in order to mark the expected position. Afterward, he returns to his neutral position and finally replicates the specificed angle. The task is performed under two experimental conditions: (\#1) without biofeedback (Nobiofeedback), (\#2) with vibrotactile biofeedback in repulsive mode (Biofeedback). Each condition consists in a serie of five successive trials. The order of presentation of the two conditions is randomized. Participants were not informed about their performances after each condition.
The smartphone is fixed on the side portion of the fibula and distally (above the malleolus). The participant wears headphones to hear the instructions from the Smartphone and uses a push button to "tag" his positions. A vibrotactile device connected via Bluetooth to the Smartphone is positioned on the ventral side of the tibia, on medial and central position. A second device is positioned at the opposite, in dorsal position (slightly caudal) on the gastrocnemius muscle. During the knee extension movement, the participant closes his eyes in order to prevent the use of a visual feedback.

At each trial, the participant starts from rest position, dangling leg. Before the first test, this position is calibrated by the participant during his first tap on the push button. He receives the setpoint (voice emitted by the Smartphone) of knee extension at 20 degrees until he hears the beep. When this audio signal is continuous, the target angle is found and the aim is to keep it in order to memorise it for further retrieving. At this moment, the participant has to push a button to mark the position. Then, when the voice command restarts, the participant returns to the rest position.

\section{Data collection and analysis}

Two dependent variables were used to assess knee joint position sense test performances:

(1) The absolute error (AE), the absolute value of the difference between the target position and the position reproduced by the participant, is a measure of the overall accuracy of the knee joint repositioning.

(2) The variable error (VE), the variance around the mean constant angular error (i.e., the average of the differences between target position and the position reproduced by the participant, paying attention to the direction of the error).

Decreased AE and VE scores indicate increased accuracy and consistency of the knee joint repositioning, respectively.

\section{Statistical analysis}

To evaluate the effect of biofeedback on knee joint proprioception sense, the two dependant variables (AE and VE) were subjected to separate two-tailed paired-samples ttests. Level of significance was set at 0.05 .

\section{E. Results}

\section{Knee joint repositioning accuracy}

As illustrated in Fig. 3 (left), analysis of the AE showed smaller values in the Biofeedback than in the Nobiofeedback condition $(\mathrm{t}=3.38, \mathrm{P}<0.01)$.

\section{Knee joint repositioning variability}

As illustrated in Fig. 3 (right), analysis of the VE showed smaller values in the Biofeedback than No-biofeedback condition $(\mathrm{t}=2.65, \mathrm{P}<0.05)$.

\section{CONCLUSION}

We have developed a Smartphone-based system for the measurement and the improvement of proprioceptive function named "iProprio". This system provides a vibrotactile biofeedback with connected bluetooth actuators 
$38^{\text {th }}$ International Conference of the IEEE Engineering in Medicine \& Biology Society, Aug. 16-19 2016, Orlando pp. $2622-2625$.
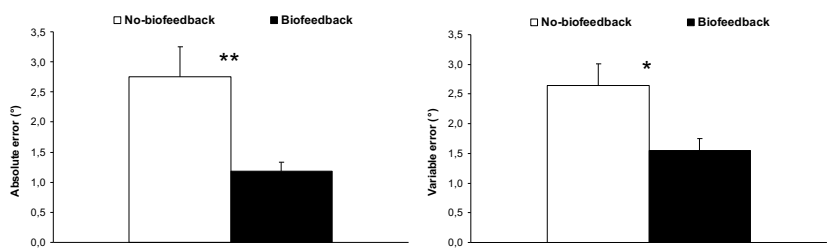

Fig. 3. Mean and standard error of mean for the absolute error (left) and variable error (right) obtained in the No-biofeedback and the Biofeedback conditions. These two experimental conditions are presented with different symbols: No-biofeedback (white bar) and Biofeedback (black bar) ${ }^{* *}$ : $\mathrm{P}<0.01, *: \mathrm{P}<0.05)$.

placed on leg in order to deliver knee joint position sense exercises. Measures of the accuracy and the variability of the knee joint position sense when the vibrotactile biofeedback was provided and when it was not were determined using absolute and variable errors.

Results of the proof-of-concept study showed reduced absolute errors (Fig. 3, left) and variable errors (Fig. 3, right) scores when the vibrotactile biofeedback was provided to the user. Taken together, these results suggest that young healthy adults were able to take advantage of vibrotactile biofeedback to increase knee joint repositioning accuracy and consistency and that repulsive biofeedback used in the present study is effective in improving the position sense at the knee joint. From a fundamental perspective in the context of sensory re-weighting process, referring the central nervous system capacity of combining information coming from different sensory cues to provide a more accurate representation of the body (e.g., [14]), the present findings suggest that the central nervous system was able to integrate an artificial biofeedback delivered through vibrotactile stimulation of the leg to improve proprioceptive acuity at the knee joint. To our knowledge, no studies on the positive effect vibrotactile biofeedback on knee joint proprioception have been published. Studies had used the Smartphone with aim of measure joint angles in the most automatic, embedded and accurate way as possible. The originality of our work is that the iProprio system is integrated in a Smartphone, and that the system was conceived to offer a specialized home-based rehabilitation training. In this purpose, protocols are entirely automated thanks to voice command and users can follow it in order to easily execute their proprioceptive exercises. iProprio is part of a tool assembly for Ambient Assisted Living which also includes the Smartphone-based audio biofeedback balance system, named "iBalance" [15]. This system requires the smartphone to be installed in a belt on posterior low back. It was designed to provide an audio biofeedback about trunk stability to the users and has proved its efficacy in improving standing balance in young healthy individuals.

Finally, this study has been conducted with young healthy individuals under normal knee neuromuscular and cognitive states. Further studies are needed to strengthen the clinical value of our approach. The effectiveness of the iProprio system used in this study is currently being assessed in older adults and knee osteoarthritis patients, known to present altered knee joint proprioception and for whom the consequences of the impairment can be dramatic (e.g., [16]).

After these studies, the perspective of this work is to be able to use such systems for autonomous rehabilitation at home for persons, in complement to the physiotherapist sessions. We believe that the present findings could have significant implications in the fields of orthopaedics and rehabilitation, for increasing, restoring and monitoriong proprioceptive functions.

\section{REFERENCES}

[1] K. R.Grob, M. S. Kuster, S. A.Higgins, D. G.Lloyd, and H.Yata, "Lack of correlation between different measurements of proprioception in the knee," J. Bone Joint Surg., vol. 84, no. 4, pp. 614-618, Apr. 2001.

[2] F. Ribeiro and J. Oliveira, "Factors Influencing Proprioception: What do They Reveal?",Biomechanics in Applications, Dr Vaclav Klika (Ed.), ISBN: 978-953-307-969-1, InTech, 2011.

[3] L. Hay, C. Bard, M. Fleury, and N. Teasdale, "Availability of visual and proprioceptive afferent messages and postural control in elderly adults," Exp. Brain Res., vol. 108, no. 1, pp. 129-139, Sep. 1995.

[4] G. C. Gauchard, C. Jeandel, A. Tessier, and P. P. Perrin, "Beneficial effect of proprioceptive physical activities on balance control in elderly human subjects," Neurosci.Lett., vol. 273, no. 2, pp. 81-84, Jul. 1999.

[5] D.J. Goble "Proprioceptive acuity assessment via joint position matching: From basic science to general practice," Phys. ther., vol. 90 no. 8, pp. 1176-84, 2010.

[6] R. L. Gajdosik, and R. W. Bohannon, "Clinical measurement of range of motion review of goniometry emphasizing reliability and validity," Phys. Ther., vol. 67, no. 12, pp. 1867-1872, 1987.

[7] E. Ozdalga, A Ozdalga, and N. Ahuja, "The smartphone in medicine: a review of current and potential use among physicians and students," J. Med. Internet Res., vol. 14, no. 5, Sep. 2012.

[8] M. N. Boulos, S. Wheeler, C. Tavares, and R. Jones, "How smartphones are changing the face of mobile and participatory healthcare: an overview, with example from eCAALYX," Biomed. Eng. Online, vol. 10, no. 1, pp. 24, Apr. 2011.

[9] M. Ockendon, and R.E. Gilbert, "Validation of a novel smartphone accelerometer-based knee goniometer," J. Knee Surg., vol. 25, no 4, pp. 341-345, Sep. 2012.

[10] F. M. Peters, R.Greeff, N. Goldstein, and C. T. Frey, "Improving acetabular cup orientation in total hip arthroplasty by using smartphone technology", J. Arthroplasty, vol. 27, no 7, pp. 13241330, 2013.

[11] S. H. Shin, D. H. Ro, O. Lee, J. H. Oh, and S. H. Kim, "Within-day reliability of shoulder range of motion measurement with a smartphone," Manual Ther., vol. 17, no 4, pp. 298-304, Feb. 2012.

[12] G. Ferriero, S. Vercelli, F. Sartorio, S. Muñoz Lasa, E. Ilieva, E. Brigatti, C. Ruella, and C. Foti., "Reliability of a smartphone-based goniometer for knee joint goniometry,” Int. J. Rehabil. Res., vol. 36, no 2, pp. 146-151, Jun. 2013.

[13] J. Lonn, A. G. Crenshaw, M. Djupsjobacka, H. Johansson , "Reliability of position sense testing assessed with a fully automated system," Clin. Physiol, vol. 20, no.1,pp. 30-37, 2000.

[14] G. A. Calvert, C. Spence, B.E. Stein (Eds.), "The Handbook of Multisensory Process," MIT Press, Cambridge, MA, 2004.

[15] C. Franco, A. Fleury, P. Y. Gumery, B. Diot, J. Demongeot, and N. Vuillerme, "iBalance-ABF: a smartphone-based audio-biofeedback balance system. Biomedical Engineering," IEEE T Bio-Med Eng, vol. 60, no. 1, pp. 211-215, 2013.

[16] J. Knoop, M. P. Steultjens, M. van der Leeden, M. van der Esch, C. A. Thorstensson, L. D. Roorda, W. F. Lems, J. Dekker, "Proprioception in knee osteoarthritis: a narrative review," Osteoarthr. Cartilage., vol. 19, no. 4, pp. 381-8, 2011.

[17] Mourcou, Q., Fleury, A., Franco, C., Klopcic, F., Vuillerme, N., "Performance evaluation of smartphone inertial sensors measurement for range of motion," Sensors, vol. 15, no. 9, pp. 23168-23187, 2015. 